International Electronic Journal of Geometry

Volume 8 No. 2 PP. 154-167 (2015) (C)IEJG

\title{
A COARSELY INVARIANT NOTION OF ROUNDNESS
}

\author{
ALEXANDER BASYROV AND MATTHEW HORAK
}

(Communicated by H. Hilmi HACISALIHOĞLU)

\begin{abstract}
We introduce the notion of "coarse roundness" for a metric space and prove that a metric space is coarsely round if and only if it coarsely embeds into a Hilbert space. Coarse roundness is a combination of the classical notions of generalized roundness and the existence of a negative kernel. It retains many of the attractive properties of both while avoiding some of the difficulties. In particular, unlike generalized roundness, coarse roundness is a coarse invariant. However, coarse roundness avoids working with sequences $\left(z_{i}\right)$ such that $\sum z_{i}=0$ as with negative kernels. We finish the paper by providing a concrete example of a metric space with bounded geometry that is coarsely round but not classically round.
\end{abstract}

\section{INTRODUCTION}

The notion of uniform embeddings was introduced by Gromov in [4] and in problems (4) and (5) of [3] as a tool for the study of properties of discrete groups such as a-T-menability and the Novikov conjecture. We use the term coarse embedding throughout this paper as it seems to be the more popular term. The idea of coarse embeddings applies to any metric space, not only those arising from the word metric on discrete groups. The question of whether or not a metric space coarsely embeds into Hilbert space has become of interest as a question in its own right. Much work has recently been done on various aspects of coarse embeddability of spaces into Banach spaces or of Banach spaces into each other $[1,8,9]$.

One reason for the recent interest in coarse embeddings into Hilbert space is the result by $\mathrm{Yu}$ [11], which implies that every discrete metric space with bounded geometry that coarsely embeds into Hilbert space satisfies the coarse Baum-Connes conjecture. Since the word metric on a finitely generated group endows the group with a discrete metric of bounded geometry, this result applies to finitely generated groups and implies that if $G$ is a finitely generated group that coarsely embeds in Hilbert space and whose classifying space has the homotopy type of a finite $\mathrm{CW}$ complex then the strong Novikov conjecture holds for $G$.

One tool in the study of coarse embeddability into Hilbert or Banach spaces is the notion of generalized roundness, which goes back to Enflo's construction in [2]

Date: Received: June 18, 2014 and Accepted: October 15, 2015.

2010 Mathematics Subject Classification. 54E40, 46B20, 46 C05.

Key words and phrases. Coarse embedding, uniform embedding, Hilbert space, roundness. 
of a countable metric space that is not uniformly homeomorphic with any subset of $L_{2}[0,1]$. Leonard, Tonge and Weston [6] clarified the relationship between generalized roundness and the notion of "negative type," or in different terminology the existence of a negative kernel. Nowak [7] has characterized the coarse embeddability of a metric space $X$ into Hilbert space in terms of the existence of a negative definite kernel on $X$ satisfying some additional properties. Thus, one may investigate the coarse embedabilty of a metric space into Hilbert space by investigating its generalized roundness. Lafont and Prassidis took this approach in [5] by carefully studying the roundness of a number of classes of finitely generated groups. They concluded the paper with the statement that the development of a coarsely invariant analog of roundness would be useful in the investigation of the BaumConnes and strong Novikov conjectures for finitely generated groups because the generalized roundness property depends strongly on the metric and hence fails to be a coarse invariant.

In this paper, we introduce the notion of "coarse roundness" and in Section 3 prove our main theorem:

Theorem 3.1. The metric space $(X, d)$ is coarsely round if and only if there exists a coarse embedding of $X$ into a Hilbert space.

Our notion of coarse roundness combines the attractive elements of negative definite kernels and generalized roundness in that it guarantees a coarse embedding into a Hilbert space. This is analogous to the fact that the existence of a negative definite kernel that is bounded by two increasing but unbounded functions guarantees a coarse embedding into Hilbert space. On the other hand, coarse roundness is established through the study of the behavior of metric on double simplices as for the generalized roundness property. However, coarse roundness is coarse invariant, which is not the case for generalized roundness.

\section{Coarse Roundness and Coarse Embeddings}

The definition of coarse roundness involves $2 n$-double simplices.

Definition 2.1. A $2 n$-double simplex in the metric space $X$ is a pair $(\mathbf{a}, \mathbf{b})$ of $n$-tuples of points $\mathbf{a}=\left(a_{1}, a_{2}, \ldots, a_{n}\right) \in X^{n}, \mathbf{b}=\left(b_{1}, b_{2}, \ldots, b_{n}\right) \in X^{n}$. The double simplex $(\mathbf{a}, \mathbf{b})$ above will be denoted by $\left[a_{i} ; b_{i}\right]_{i=1}^{n}$. We note that the points $a_{i}$ and $b_{i}$ need not be distinct.

The following notion of coarse roundness takes inspiration from the generalized roundness of [2]. We show in Section 3 that a metric space is coarsely round if and only if it is coarsely embeddable into a Hilbert space.

Definition 2.2. The metric space $(X, d)$ is coarsely round if there exists a function $\sigma: X \times X \rightarrow[0, \infty)$ and a pair of non-decreasing functions $\rho_{1}, \rho_{2}:[0, \infty) \rightarrow[0, \infty)$ with $\lim _{t \rightarrow \infty} \rho_{1}(t)=\infty$ such that,

(1) $\sigma(x, x)=0$ for all $x \in X$,

(2) $\rho_{1}(d(x, y)) \leq \sigma(x, y) \leq \rho_{2}(d(x, y))$ for all $x, y \in X$,

(3) For any $2 n$-double simplex $\left[a_{i} ; b_{i}\right]_{i=1}^{n}$ in $X$, we have

$$
\sum_{1 \leq i<j \leq n}\left(\sigma\left(a_{i}, a_{j}\right)+\sigma\left(b_{i}, b_{j}\right)\right) \leq \sum_{1 \leq i, j \leq n} \sigma\left(a_{i}, b_{j}\right) .
$$


We refer to the function $\sigma$ as a coarse roundness function for $X$, and the functions $\rho_{i}$ as the controlling functions for $\sigma$. For conciseness, we say that $\sigma$ is a roundness function for $X$ with controlling functions $\rho_{1} \leq \rho_{2}$. Note that whenever we refer to controlling functions, we always assume that the functions are nondecreasing functions with domain and range $[0, \infty)$ and $\lim _{t \rightarrow \infty} \rho_{1}(t)=\infty$.

The following lemma states that roundness functions are symmetric. This property is a manifestation of the fact that the roundness inequality (3) above is a very strong one since the proof of symmetry relies upon the roundness inequality alone and not on the existence of bounding controlling functions.

Lemma 2.1. Let $(X, d)$ coarsely round metric space with roundness function $\sigma$. Then $\sigma$ is symmetric. That is, $\sigma(x, y)=\sigma(y, x)$ for any $x, y \in X$.

Proof. Fix $x, y \in X$. We consider the 4 -double simplex $\left[a_{i} ; b_{i}\right]_{i=1}^{2}$ given by $a_{1}=$ $x, a_{2}=y, b_{1}=x, b_{2}=y$. For the chosen 4-double simplex, the roundness inequality (3) from Definition 2.2 is

$$
\sigma(x, y)+\sigma(x, y) \leq \sigma(x, x)+\sigma(x, y)+\sigma(y, x)+\sigma(y, y)
$$

which reduces to $\sigma(x, y) \leq \sigma(y, x)$.

Considering another 4-double simplex $\left[a_{i} ; b_{i}\right]_{i=1}^{2}$ with $a_{1}=y, a_{2}=x, b_{1}=y, b_{2}=$ $x$ we similarly arrive at $\sigma(y, x) \leq \sigma(x, y)$, completing the proof of lemma.

We recall the definition of a coarse embedding of metric spaces, as given in [10].

Definition 2.3. Let $\left(X, d_{X}\right)$ and $\left(Y, d_{Y}\right)$ be metric spaces. A function $f: X \rightarrow Y$ is a coarse embedding of $X$ into $Y$ if there are non-decreasing functions $\rho_{1}, \rho_{2}$ : $[0, \infty) \rightarrow[0, \infty)$ with $\lim _{t \rightarrow \infty} \rho_{1}(t)=\infty$ such that for all $a, b \in X$

$$
\rho_{1}\left(d_{X}(a, b)\right) \leq d_{Y}(f(a), f(b)) \leq \rho_{2}\left(d_{X}(a, b)\right) .
$$

Again, we refer to the functions $\rho_{1}$ and $\rho_{2}$ as controlling functions for $f$ and we say that $f$ is a coarse embedding of $X$ into $Y$ controlled by $\rho_{1} \leq \rho_{2}$. Again, whenever we refer to controlling functions for a coarse embedding function, we always assume that the functions are nondecreasing functions with domain and range $[0, \infty)$ and $\lim _{t \rightarrow \infty} \rho_{1}(t)=\infty$.

We note that Definitions 2.2 and 2.3 give two different usages for the term controlling function. The notions are very similar, and context will always make clear which usage is meant in every particular instance.

Theorem 2.1. Let $\left(X, d_{X}\right)$ be a metric space and $\left(Y, d_{Y}\right)$ be a coarsely round metric space. If $X$ coarsely embeds into $Y$ then $X$ is coarsely round.

Proof. Choose a roundness function $\sigma: Y \times Y \rightarrow[0, \infty)$ for $Y$ with controlling functions $\rho_{1} \leq \rho_{2}$. Also choose a coarse embedding $f: X \rightarrow Y$ with controlling functions $\alpha_{1} \leq \alpha_{2}$. Define the function $\sigma^{\prime}: X \times X \rightarrow[0, \infty)$ by $\sigma^{\prime}(x, y)=\sigma(f(x), f(y))$. Clearly $\sigma^{\prime}(x, x)=0$, so $\sigma^{\prime}$ satisfies the first condition of Definition 2.2 of coarse roundness.

Now, $\rho_{i}$ and $\alpha_{i}$ are nondecreasing functions for $i=1,2$. Therefore, $\rho_{i} \circ \alpha_{i}$ are nondecreasing functions for $i=1,2$ as well. Also $\lim _{t \rightarrow \infty} \rho_{1} \circ \alpha_{1}(t)=\infty$ since this is true of both $\alpha_{1}$ and $\rho_{1}$. We also have,

$$
\begin{aligned}
& \rho_{1} \circ \alpha_{1} \circ d_{X}(x, y) \leq \rho_{1} \circ d_{Y}(f(x), f(y)) \leq \sigma(f(x), f(y))=\sigma^{\prime}(x, y)= \\
& \sigma(f(x), f(y)) \leq \rho_{2} \circ d_{Y}(f(x), f(y)) \leq \rho_{2} \circ d_{Y}(f(x), f(y)) \leq \rho_{2} \circ \alpha_{2} \circ d_{X}(x, y) .
\end{aligned}
$$


Therefore $\sigma^{\prime}, \rho_{1} \circ \alpha_{1}$ and $\rho_{2} \circ \rho_{2}$ satisfy the second condition of Definition 2.2.

Finally, consider the $2 n$-double simplex $\left[a_{i} ; b_{i}\right]_{i=1}^{n}$ in $X$. Now, $\left[f\left(a_{i}\right) ; f\left(b_{i}\right)\right]_{i=1}^{n}$ is a $2 n$-double simplex in $Y$. Since $\sigma$ is a coarse roundness function for $Y$, we have

$$
\begin{aligned}
\sum_{1 \leq i<j \leq n}\left(\sigma^{\prime}\left(a_{i}, a_{j}\right)+\sigma^{\prime}\left(b_{i}, b_{j}\right)\right) & =\sum_{1 \leq i<j \leq n}\left(\sigma\left(f\left(a_{i}\right), f\left(a_{j}\right)\right)+\sigma\left(f\left(b_{i}\right), f\left(b_{j}\right)\right)\right) \\
& \leq \sum_{1 \leq i, j \leq n} \sigma\left(f\left(a_{i}\right), f\left(b_{j}\right)\right)=\sum_{1 \leq i, j \leq n} \sigma^{\prime}\left(a_{i}, b_{j}\right) .
\end{aligned}
$$

Therefore $\sigma^{\prime}, \rho_{1} \circ \alpha_{1}$ and $\rho_{2} \circ \rho_{2}$ satisfy the third condition of Definition 2.2 so $\sigma^{\prime}$ is a coarse roundness function for $X$ with controlling functions $\rho_{1} \circ \alpha_{1} \leq \rho_{2} \circ \alpha_{2}$, proving that $X$ is coarsely round.

\section{Coarse Embeddings into Hilbert Space}

One common tool in the study of embeddings into Hilbert space and other metric spaces is the idea of a negative definite kernel, defined below.

Definition 3.1. Let $X$ be a set. A function $k: X \times X \rightarrow \mathbb{R}$ is a negative definite kernel if

(1) For all $x, y \in X$, we have $k(x, y)=k(y, x)$ and

(2) If $x_{1}, x_{2}, \ldots, x_{n} \in X$ and $\lambda_{1}, \lambda_{2}, \ldots, \lambda_{n} \in \mathbb{R}$ with $\sum \lambda_{i}=0$ then

$$
\sum \lambda_{i} \lambda_{j} k\left(x_{i}, x_{j}\right) \leq 0
$$

The negative definite kernel $k$ is called normalized if additionally

3. $k(x, x)=0$ for all $x \in X$.

We need the following lemma, which is well-known and follows directly from the definitions.

Lemma 3.1. If $\mathcal{H}$ is a real Hilbert space then the function $k(x, y)=\|x-y\|^{2}$ is a normalized negative definite kernel on $\mathcal{H}$.

The next lemma is proved in [10] in the context of coarse spaces whose coarse structure is not necessarily given by a metric. We rephrase it as in [7] in way more suited to the context of coarsely round metric spaces and provide a proof of this version. A good sketch of the proof of this lemma can also be found in [7].

Lemma 3.2. If $k$ is a normalized negative definite kernel on the metric space $(X, d)$ that is bounded by the nondecreasing functions $\rho_{1}, \rho_{2}:[0, \infty) \rightarrow[0, \infty)$ in the sense that $\rho_{1}(d(x, y)) \leq k(x, y) \leq \rho_{2}(d(x, y))$ and if $\lim _{t \rightarrow \infty} \rho_{1}(t)=\infty$ then $X$ coarsely embeds in a Hilbert space.

Proof. Define $W$ to be the real vector space of all finitely supported functions $f: X \rightarrow \mathbb{R}$ such that $\sum_{X} f(x)=0$. The formula,

$$
\langle f, g\rangle=-\frac{1}{2} \sum_{x, y} k(x, y) f(x) g(y)
$$


clearly defines a bilinear form on $W$. We claim that $\langle\cdot, \cdot\rangle$ is positive semi-definite. To prove this, let $f \in W$. Since $k$ is negative definite,

$$
\sum_{x, y \in \operatorname{Dom}(f)} k(x, y) f(x) f(y) \leq 0 .
$$

Therefore,

$$
\langle f, f\rangle=-\frac{1}{2} \sum_{x, y \in \operatorname{Dom}(f)} k(x, y) f(x) f(y) \geq 0 .
$$

Let $W_{0}=\{f \in W \mid\|f\|=0\}$. Now, $W_{0}$ is a subspace of $W$ and we define $\mathcal{H}_{0}=W / W_{0}$. The positive semi-definite bilinear form $\langle\cdot, \cdot\rangle$ on $W$ induces a positive definite bilinear form on $\mathcal{H}_{0}$ with respect to which $\mathcal{H}_{0}$ is a Hilbert space.

Arbitrarily fix a basepoint $p \in X$. Define $\phi: X \rightarrow \mathcal{H}_{0}$ by,

$$
\phi(x)=\delta_{x}-\delta_{p},
$$

where $\delta_{z}: X \rightarrow \mathbb{R}$ is the function taking value 1 at $z$ and 0 elsewhere. Now, for $x, y \in X$, we have

$$
d_{\mathcal{H}}(\phi(x), \phi(y))=\|\phi(x)-\phi(y)\|=\left\|\delta_{x}-\delta_{y}\right\|=\sqrt{\left\langle\delta_{x}-\delta_{y}, \delta_{x}-\delta_{y}\right\rangle}=\sqrt{k(x, y)} .
$$

Therefore,

$$
\sqrt{\rho_{1}(d(x, y))} \leq d_{\mathcal{H}}(\phi(x), \phi(y)) \leq \sqrt{\rho_{2}(d(x, y))} .
$$

Since $\rho_{1}$ and $\rho_{2}$ are nondecreasing functions with domain $[0, \infty)$, the same is true of $\sqrt{\rho_{1}}$ and $\sqrt{\rho_{2}}$. Also, $\lim _{t \rightarrow \infty} \sqrt{\rho_{1}(t)}=\infty$ since $\lim _{t \rightarrow \infty} \rho_{1}(t)=\infty$. Therefore, $\phi$ defines a coarse embedding of $X$ into the Hilbert space $\mathcal{H}_{0}$ controlled by $\sqrt{\rho_{1}} \leq \sqrt{\rho_{2}}$.

The connection of roundness to coarse embeddings into a Hilbert space is through the connection between roundness and negative kernels. This connection is given explicitly in Lemmas 3.3 and 3.4 below. Versions of these results for generalized (non-coarse) roundness are known and proved in [6] as Theorems 2.2 and 2.4. The proofs in the setting of coarse roundness are similar to those for generalized roundness, but slight modifications are required to deal with the fact that a coarse roundness function need not be a function of the metric. The fully modified proofs are presented here.

Lemma 3.3. Let $(X, d)$ be a metric space with coarse roundness function $\sigma$. For all $n \in \mathbb{N}$ and all finite sequences $x_{1}, x_{2}, \ldots, x_{n}$ of points in $X$ and all collections of weights

$$
w_{1}, w_{2}, \ldots, w_{n}, s_{1}, s_{2}, \ldots, s_{n} \geq 0
$$

satisfying $\sum_{j=1}^{n} w_{j}=\sum_{j=1}^{n} s_{j}=1$, we have

$$
\sum_{i, j=1}^{n} \sigma\left(x_{i}, x_{j}\right)\left(w_{i}-s_{i}\right)\left(w_{j}-s_{j}\right) \leq 0 .
$$

Proof. We first note that by the symmetry of $\sigma$, Inequality (3.1) is equivalent to,

$$
\sum_{1 \leq i, j \leq n}\left(w_{i} w_{j}+s_{i} s_{j}\right) \sigma\left(x_{i}, x_{j}\right) \leq 2 \sum_{1 \leq i, j \leq n} w_{i} s_{j} \sigma\left(x_{i}, x_{j}\right) .
$$

We now prove Inequality (3.2). 
To begin, let $n \in \mathbb{N}$ and let $x_{1}, x_{2}, \ldots, x_{n}$ be a sequence of points in $X$. Let $N \geq n$ and suppose that $l_{1}, l_{2}, \ldots, l_{n}, m_{1}, m_{2}, \ldots, m_{n}$ are nonnegative integers satisfying $\sum \frac{l_{j}}{N}=\sum \frac{m_{j}}{N}=1$. Construct a $2 N$ double simplex $\left[a_{i} ; b_{i}\right]_{i=1}^{N}$ as follows. Set,

$$
a_{i}= \begin{cases}x_{1} & \text { if } 1 \leq i \leq l_{1} \\ x_{2} & \text { if } l_{1}<i \leq l_{1}+l_{2}, \\ x_{3} & \text { if } l_{1}+l_{2}<i \leq l_{1}+l_{2}+l_{3}, \\ \vdots & \vdots \\ x_{n} & \text { if } l_{1}+l_{2}+\cdots+l_{n-1}<i \leq N .\end{cases}
$$

And set,

$$
b_{i}= \begin{cases}x_{1} & \text { if } 1 \leq i \leq m_{1}, \\ x_{2} & \text { if } m_{1}<i \leq m_{1}+m_{2}, \\ x_{3} & \text { if } m_{1}+m_{2}<i \leq m_{1}+m_{2}+m_{3}, \\ \vdots & \vdots \\ x_{n} & \text { if } m_{1}+l_{2}+\cdots+m_{n-1}<i \leq N .\end{cases}
$$

Condition (3) from Definition 2.2 of coarse roundness for this double simplex becomes,

$$
\sum_{1 \leq i<j \leq N}\left(\sigma\left(a_{i}, a_{j}\right)+\sigma\left(b_{i}, b_{j}\right)\right) \leq \sum_{1 \leq i, j \leq N} \sigma\left(a_{i}, b_{j}\right) .
$$

Analyzing the left hand side of (3.3), recalling that $\sigma(x, x)=0$ and that $\sigma$ is symmetric, we find that,

$$
\begin{aligned}
\sum_{1 \leq i<j \leq N}\left(\sigma\left(a_{i}, a_{j}\right)+\sigma\left(b_{i}, b_{j}\right)\right)= & \sum_{1 \leq i<j \leq n}\left(l_{i} l_{j}+m_{i} m_{j}\right) \sigma\left(x_{i}, x_{j}\right)= \\
& \frac{1}{2} \sum_{1 \leq i, j \leq n}\left(l_{i} l_{j}+m_{i} m_{j}\right) \sigma\left(x_{i}, x_{j}\right) .
\end{aligned}
$$

Similarly, the right hand side becomes,

$$
\sum_{1 \leq i, j \leq N} \sigma\left(a_{i}, b_{j}\right)=\sum_{1 \leq i, j \leq n} l_{i} m_{j} \sigma\left(x_{i}, x_{j}\right) .
$$

Therefore,

$$
\sum_{1 \leq i, j \leq n} \sigma\left(x_{i}, x_{j}\right)\left(\frac{l_{i}}{N} \frac{l_{j}}{N}+\frac{m_{i}}{N} \frac{m_{j}}{N}\right) \leq 2 \sum_{1 \leq i, j \leq n} \sigma\left(x_{i}, x_{j}\right)\left(\frac{l_{i}}{N} \frac{m_{j}}{N}\right) .
$$

Now let, $w_{1}, w_{2}, \ldots, w_{n}, s_{1}, s_{2}, \ldots, s_{n} \geq 0$ satisfy $\sum_{j=1}^{n} w_{j}=\sum_{j=1}^{n} s_{j}=1$. The set

$$
A=\left\{\left(\frac{k_{1}}{N}, \frac{k_{2}}{N}, \ldots, \frac{k_{n}}{N}\right) \mid N, k_{i} \in \mathbb{N}, N \geq n \text { and } \sum \frac{k_{i}}{N}=1\right\}
$$

is dense in

$$
B=\left\{\left(t_{1}, t_{2}, \ldots, t_{n}\right) \mid t_{i} \in \mathbb{R}, t_{i} \geq 0 \text { and } \sum t_{i}=1\right\},
$$

and we have just shown Inequality (3.2) to be true on all pairs of $n$-tuples of weights in $A$. Selecting sequences of $n$-tuples of weights in $A$ converging to $\left(w_{1}, w_{2}, \ldots, w_{n}\right)$ and $\left(s_{1}, s_{2}, \ldots, s_{n}\right)$, taking limits and observing that as functions of the weights 
both sides of Inequality (3.2) are continuous proves that the inequality is true for weights $w_{1}, w_{2}, \ldots, w_{n}$ and $s_{1}, s_{2}, \ldots, s_{n}$ as required.

Lemma 3.4. Let $\sigma$ be a coarse roundness function on $X$. Then, $\sigma$ is a normalized negative kernel on $X$.

Proof. First, let $x_{1}, x_{2}, \ldots, x_{n} \in X$ and let $\lambda_{1}, \lambda_{2}, \ldots, \lambda_{n} \in \mathbb{R}$ with $\sum \lambda_{i}=0$. Let $M=\sum_{\lambda_{i} \geq 0} \lambda_{i}$. If $\lambda_{i} \geq 0$ set $w_{i}=\frac{\lambda_{i}}{M}$ and $s_{i}=0$ if $\lambda_{i}<0$ set $w_{i}=0$ and $s_{i}=-\frac{\lambda_{i}}{M}$. By Lemma 3.3,

$$
\sum_{i, j=1}^{n} \sigma\left(x_{i}, x_{j}\right)\left(w_{i}-s_{i}\right)\left(w_{j}-s_{j}\right) \leq 0
$$

But, $w_{i}-s_{i}=\frac{\lambda_{i}}{M}$ for all $i$. Therefore,

so,

$$
\sum_{1 \leq i, j \leq n} \frac{\lambda_{i} \lambda_{j}}{M^{2}} \sigma\left(x_{i}, x_{j}\right) \leq 0
$$

$$
\sum_{1 \leq i, j \leq n} \lambda_{i} \lambda_{j} \sigma\left(x_{i}, x_{j}\right) \leq 0
$$

Thus, $\sigma$ satisfies Condition (2) of Definition 3.1 of a negative kernel. Conditions (1) and (3) are satisfied by the fact that $\sigma$ is symmetric by Lemma 2.1 and that $\sigma(x, x)=0$ by Condition (1) of Definition 2.2 of coarse roundness. Therefore, $\sigma$ is a normalized negative kernel on $X$.

We are now in the position to prove our main theorem.

Theorem 3.1. The metric space $(X, d)$ is coarsely round if and only if there exists a coarse embedding of $X$ into a Hilbert space.

Proof. First assume that $X$ coarsely embeds into the Hilbert space $\mathcal{H}$ under coarse embedding $f: X \rightarrow \mathcal{H}$ controlled by $\alpha_{1} \leq \alpha_{2}$. Define $\sigma: X \times X \rightarrow \mathbb{R}$ by $\sigma(x, y)=\|f(x)-f(y)\|^{2}$. Now, $\sigma$ clearly satisfies Condition (1) of Definition 2.2 of coarse roundness. By Lemma 3.1, $\sigma$ is a normalized negative definite kernel on $X$. Since $f$ is controlled by $\alpha_{1}$ and $\alpha_{2}$, we have

$$
\alpha_{1}(d(x, y)) \leq \sqrt{\sigma(x, y)} \leq \alpha_{2}(d(x, y)) .
$$

Therefore,

$$
\left[\alpha_{1}(d(x, y))\right]^{2} \leq \sigma(x, y) \leq\left[\alpha_{2}(d(x, y))\right]^{2} .
$$

Since $\left[\alpha_{1}\right]^{2} \leq\left[\alpha_{2}\right]^{2}$ are nondecreasing functions with domain $[0, \infty)$ with $\lim _{t \rightarrow \infty} \alpha_{1}(t)=$ $\infty$, they are controlling functions for $\sigma$. So, Condition (2) of the definition of coarse roundness is satisfied by $\sigma$ and the controlling functions $\left[\alpha_{1}\right]^{2} \leq\left[\alpha_{2}\right]^{2}$.

Consider the $2 n$ double simplex $\left[a_{i} ; b_{i}\right]_{i=1}^{n}$ in $X$. Set $\epsilon_{i}=1, \delta_{i}=-1$ for $i=1 \ldots n$. Since $\sigma$ is a negative kernel on $X$, we have,

$$
\sum_{i, j=n}^{n} \epsilon_{i} \epsilon_{j} \sigma\left(b_{i}, b_{j}\right)+\sum_{i, j=n}^{n} \delta_{i} \delta_{j} \sigma\left(a_{i}, a_{j}\right)+\sum_{i, j=n}^{n} \epsilon_{i} \delta_{j} \sigma\left(a_{i}, b_{j}\right)+\sum_{i, j=n}^{n} \delta_{i} \epsilon_{j} k\left(b_{i}, a_{j}\right) \leq 0 .
$$

Therefore,

$$
\sum_{i, j=1}^{n} \sigma\left(a_{i}, a_{j}\right)+\sum_{i, j=1}^{n} \sigma\left(b_{i}, b_{j}\right) \leq \sum_{i, j=1}^{n} \sigma\left(a_{i}, b_{j}\right)+\sum_{i, j=1}^{n} \sigma\left(b_{i}, a_{j}\right) .
$$


But $\sigma$ is symmetric so on the right hand side of the inequality we have,

$$
\sum_{i, j=1}^{n} \sigma\left(a_{i}, b_{j}\right)+\sum_{i, j=1}^{n} \sigma\left(b_{i}, a_{j}\right)=2 \sum_{i, j=1}^{n} \sigma\left(a_{i}, b_{j}\right) .
$$

And on the left hand side,

$$
\sum_{i, j=1}^{n} \sigma\left(a_{i}, a_{j}\right)+\sum_{i, j=1}^{n} \sigma\left(b_{i}, b_{j}\right)=2 \sum_{1 \leq i<j \leq n}\left(\sigma\left(a_{i}, a_{j}\right)+\sigma\left(b_{i}, b_{j}\right)\right) .
$$

Therefore,

$$
\sum_{1 \leq i<j \leq n}\left(\sigma\left(a_{i}, a_{j}\right)+\sigma\left(b_{i}, b_{j}\right)\right) \leq \sum_{i, j=1}^{n} \sigma\left(a_{i}, b_{j}\right),
$$

so $\sigma$ satisfies Condition (3) of Definition 2.2 of coarse roundness. Hence $X$ is coarsely round.

Now assume that $X$ is coarsely round with coarse roundness function $\sigma$ bounded by functions $\rho_{1}$ and $\rho_{2}$ with $\lim _{t \rightarrow \infty} \rho_{1}(t)=\infty$. By Lemma 2.1, $\sigma$ is a symmetric. By Lemma 3.4, $\sigma$ is a normalized negative kernel on $X$ controlled by $\rho_{1} \leq \rho_{2}$. Therefore, by Lemma 3.2, $X$ coarsely embeds in a Hilbert space.

\section{EXAMPLES}

In this section we present concrete examples of metric spaces that have generalized roundness 0 (that is, they have generalized roundness $q$ for only $q=0$ ) but which are coarsely round. We note that examples of such spaces are known. For example, those groups described in Proposition 5.8 of [5] have generalized roundness 0 but do coarsely embed in a Hilbert space. However, the fact that these groups have generalized roundness 0 relies on the non-trivial fact that every Kazhdan group has generalized roundness 0 . The examples we present below have elementary proofs of both their coarse roundness or coarse embeddability into Hilbert space and the fact their generalized roundness is maximally 0 .

The examples we present increase in complexity as the number of their desirable properties increase. Recall that a discrete metric space is said to be locally finite if any given ball of finite radius contains only finitely many elements. A stronger condition is that of having bounded geometry, which means that for every $n \in \mathbb{N}$, there is a constant $M_{n}$ such that any ball of radius at most $n$ contains at most $M_{n}$ points. Since the word metric on a finitely generated group has bounded geometry, our final goal is to present a space of bounded geometry that has generalized roundness only 0 , but that is coarsely round. This is fairly complicated and we do this in Example 3. Example 2 is a space that does not have bounded geometry, but whose metric is locally finite. Example 1 is the least complicated example and forms the basis of Examples 2 and 3, but it is not a locally finite space.

4.1. Example 1. Consider the set

$$
F=\{f=(x, n): x \in \mathbb{Z}, n \in \mathbb{N}\}
$$

with natural projection $\tau: F \rightarrow \mathbb{Z}$

$$
\tau:(x, n) \mapsto x
$$


and function $d: F \times F \rightarrow \mathbb{R}$

$$
d\left(f_{1}, f_{2}\right)= \begin{cases}\left|x_{2}-x_{1}\right|, & \text { if } x_{1} \neq x_{2} \\ 2, & \text { if } x_{1}=x_{2}, \quad n_{1} \neq n_{2} \\ 0, & \text { if }\left(x_{1}, n_{1}\right)=\left(x_{2}, n_{2}\right)\end{cases}
$$

Lemma 4.1. Function d defined by (4.1) is a metric on F.

Proof. It is obvious that the function defined in (4.1) is non-negative, and symmetric. We need to verify that $d$ satisfies the triangle inequality,

$$
d\left(f_{1}, f_{2}\right)+d\left(f_{2}, f_{3}\right) \geq d\left(f_{1}, f_{3}\right)
$$

for all $f_{1}, f_{2}, f_{3} \in F$.

We observe that the inequality (4.2) is obvious if $\tau\left(f_{1}\right)=\tau\left(f_{2}\right)=\tau\left(f_{3}\right)$. In the case $\tau\left(f_{1}\right), \tau\left(f_{2}\right), \tau\left(f_{3}\right)$ are all distinct, inequality (4.2) reduces to a triangle inequality on $\mathbb{Z}^{2}$ with $l_{1}$ norm. The only non-trivial situation is when exactly two of $f_{1}, f_{2}, f_{3}$ have the same projections. We consider the 3 possible cases:

- If $\tau\left(f_{1}\right)=\tau\left(f_{2}\right) \neq \tau\left(f_{3}\right)$, we have $d\left(f_{1}, f_{2}\right)=2$ and $d\left(f_{2}, f_{3}\right)=d\left(f_{1}, f_{3}\right)$ which reduces (4.2) to a trivial inequality

$$
2 \geq 0 \text {. }
$$

- The case $\tau\left(f_{1}\right) \neq \tau\left(f_{2}\right)=\tau\left(f_{3}\right)$ is similar to the previous one.

- If $\tau\left(f_{1}\right)=\tau\left(f_{3}\right) \neq \tau\left(f_{2}\right)$, we have $d\left(f_{1}, f_{3}\right)=2$ and $d\left(f_{1}, f_{2}\right)=d\left(f_{2}, f_{3}\right) \geq 1$ which results in

$$
d\left(f_{1}, f_{2}\right)+d\left(f_{2}, f_{3}\right) \geq 2=d\left(f_{1}, f_{3}\right) .
$$

Informally, we think of the space $F$ as illustrated below:

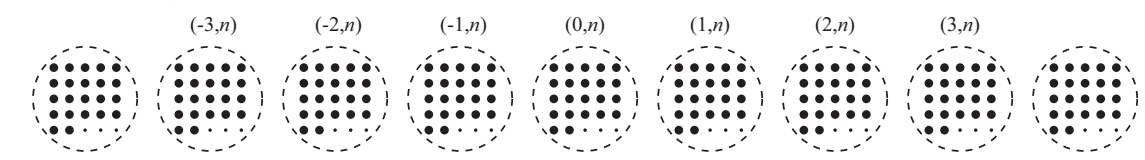

That is, the space $F$ is organized into collections of points according to the first coordinate; the distance between points in the same collection is 2 units; the distance between points in different collections is equal to the absolute value of the difference of the first coordinates.

We recall the definition of generalized roundness given, for example, in [6].

Definition 4.1. Metric space $(X, d)$ has generalized roundness $q \geq 0$ if for any $2 n$-double simplex $\left[a_{i} ; b_{i}\right]_{i=1}^{n}$ in $X$ the following inequality holds:

$$
\sum_{1 \leq i<j \leq n}\left[d^{q}\left(a_{i}, a_{j}\right)+d^{q}\left(b_{i}, b_{j}\right)\right] \leq \sum_{i, j=1}^{n} d^{q}\left(a_{i}, b_{j}\right) .
$$

We observe that any metric space $X$ has generalized roundness 0 since the left hand side of the roundness inequality has fewer terms than the right hand side. It is customary to say that a space $X$ is round if it has generalized roundness $q$ for some strictly positive $q$; otherwise the space is called not round.

Lemma 4.2. The space $(F, d)$ is not round. That is $(F, d)$ has generalized roundness $q$ only for $q=0$. 
Proof. We consider $2 n$-double simplex $\left[a_{i} ; b_{i}\right]_{i=1}^{n}$ with

$$
a_{i}=(0, i), \quad b_{i}=(1, i) .
$$

Observe that $d\left(a_{i}, a_{j}\right)=2, d\left(b_{i}, b_{j}\right)=2$ but $d\left(a_{i}, b_{j}\right)=1$. If space $(F, d)$ has generalized roundness $q \geq 0$, then the following inequality must be satisfied:

$$
\sum_{1 \leq i<j \leq n}\left[d^{q}\left(a_{i}, a_{j}\right)+d^{q}\left(b_{i}, b_{j}\right)\right] \leq \sum_{i, j=1}^{n} d^{q}\left(a_{i}, b_{j}\right)
$$

which in our case is $n(n-1) \cdot 2^{q} \leq n^{2}$. Resolving the inequality for $q$ we arrive at

$$
q \leq \log _{2}[n /(n-1)] .
$$

Since $\lim _{n \rightarrow \infty} \log _{2}[n /(n-1)]=0$ we immediately see that $q=0$ so $(F, d)$ has generalized roundness only 0 .

Lemma 4.3. The space $(F, d)$ is coarsely round with roundness function

$$
\sigma\left(f_{1}, f_{2}\right)=\left(x_{2}-x_{1}\right)^{2}=\left\|\tau\left(f_{2}\right)-\tau\left(f_{1}\right)\right\|^{2}
$$

that is controlled by

$$
\rho_{1}(t)=\left\{\begin{array}{ll}
0, & \text { if } 0 \leq t \leq 2 \\
(t-2)^{2}, & \text { if } t>2
\end{array} \quad \text { and } \quad \rho_{2}(t)=t^{2}\right.
$$

Proof. It is clear that $\sigma$ is a roundness function.

To obtain the bounding functions, we observe that the following holds for all $f_{1}$ and $f_{2}$,

$$
\sqrt{\sigma\left(f_{1}, f_{2}\right)}=\left|x_{2}-x_{1}\right| \leq d\left(f_{1}, f_{2}\right) \leq\left|x_{2}-x_{1}\right|+2 \leq \sqrt{\sigma\left(f_{1}, f_{2}\right)}+2
$$

hence the left half of the inequality implies $\sigma\left(f_{1}, f_{2}\right) \leq\left[d\left(f_{1}, f_{2}\right)\right]^{2}$, and the upper bounding function $\rho_{2}(t)=t^{2}$ is established. Using the right half of the inequality, we obtain $\sqrt{\sigma\left(f_{1}, f_{2}\right)} \geq d\left(f_{1}, f_{2}\right)-2$, and the lower bounding function

$$
\rho_{1}(t)= \begin{cases}0, & \text { if } 0 \leq t \leq 2 \\ (t-2)^{2}, & \text { if } t>2\end{cases}
$$

is established.

We summarize some of the properties of the space $F$ in the following proposition

Proposition 4.1. Metric space $(F, d)$ is an example of a space with the following properties:

(i) $F$ is a coarsely round metric space,

(ii) F coarsely embeds into a Hilbert space,

(iii) F has generalized roundness 0 ,

(iv) $F$ is not a locally finite space,

It is easy to see that the natural projection $\tau: F \rightarrow \mathbb{Z} \subset \mathbb{R}$ defines an explicit coarse embedding of $F$ into Hilbert space $\mathbb{R}$. The coarse roundness function $\sigma$ of this example is defined exactly according to the definition used in the proof of Theorem 3.1, concretely illustrating the equivalence of coarse embedding and coarse roundness. 
4.2. Example 2. We observe that space $F$ from previous example contains a locally finite subspace,

$$
F_{1}=\left\{f=(x, n) \in F: 1 \leq n \leq x^{2}+1\right\} .
$$

We observe that space $F_{1}$ is a locally finite metric space that has generalized roundness 0 and is coarsely round.

4.3. Example 3. Consider the set

$$
E=\left\{e=(g, x, n): g \in \mathbb{N}, x \in\{0,1\}, n \in\left\{1,2, \ldots, g^{2}\right\}\right\}
$$

with two natural projection functions, $\zeta: E \rightarrow \mathbb{Z}^{2}$

$$
\zeta:(g, x, n) \mapsto(g, x)
$$

and $\eta: E \rightarrow \mathbb{N}$

$$
\eta(g, x, n) \mapsto g
$$

Define function $d: E \times E \rightarrow[0,+\infty)$ by

$$
d\left(e_{1}, e_{2}\right)= \begin{cases}0, & \text { if } e_{1}=e_{2}, \\ 2 g_{1}, & \text { if } \zeta\left(e_{1}\right)=\zeta\left(e_{2}\right), e_{1} \neq e_{2}, \\ g_{1}, & \text { if } \eta\left(e_{1}\right)=\eta\left(e_{2}\right), \zeta\left(e_{1}\right) \neq \zeta\left(e_{2}\right), \\ \sum_{j=\max \left(g_{1}, g_{2}\right)} j^{2}, & \text { if } \eta\left(e_{1}\right) \neq \eta\left(e_{2}\right) .\end{cases}
$$

Lemma 4.4. Function d defined by (4.3) is a metric on E.

Proof. The proof is similar to the proof of Lemma 4.1. From the definition of the function it is clear that the only property of a metric that is in question is the triangle inequality.

We consider three points $e_{1}, e_{2}, e_{3} \in E$, and we wish to demonstrate that

$$
d\left(e_{1}, e_{2}\right)+d\left(e_{2}, e_{3}\right) \geq d\left(e_{1}, e_{3}\right)
$$

holds true. We observe that the inequality (4.4) is symmetric with respect to $e_{1}$ and $e_{3}$, so without loss of generality we assume that $\eta\left(e_{1}\right) \leq \eta\left(e_{3}\right)$. We consider several cases that cover all possibilities of a general situation.

(I) All of $\eta\left(e_{1}\right), \eta\left(e_{2}\right), \eta\left(e_{3}\right)$ are distinct. This leaves us with the following three cases for the value of $\eta\left(e_{2}\right)$.

(a) If $\eta\left(e_{2}\right)<\eta\left(e_{1}\right)<\eta\left(e_{3}\right)$, then the definition of $d$ implies $d\left(e_{2}, e_{3}\right)>$ $d\left(e_{1}, d_{3}\right)$ and inequality (4.4) holds true.

(b) If $\eta\left(e_{1}\right)<\eta\left(e_{2}\right)<\eta\left(e_{3}\right)$, then the definition of $d$ implies $d\left(e_{1}, e_{2}\right)+$ $d\left(e_{2}, e_{3}\right)=d\left(e_{1}, d_{3}\right)$ and inequality (4.4) holds true.

(c) If $\eta\left(e_{1}\right)<\eta\left(e_{3}\right)<\eta\left(e_{2}\right)$, then the definition of $d$ implies $d\left(e_{1}, e_{2}\right)>$ $d\left(e_{1}, d_{3}\right)$ and inequality (4.4) holds true.

(II) Exactly two of $\eta\left(e_{1}\right), \eta\left(e_{2}\right), \eta\left(e_{3}\right)$ are the same. The assumption $\eta\left(e_{1}\right) \leq$ $\eta\left(e_{3}\right)$, leaves the following two possibilities.

(a) If $\eta\left(e_{1}\right)=\eta\left(e_{2}\right)<\eta\left(e_{3}\right)$, then the definition of $d$ implies that $d\left(e_{2}, e_{3}\right)=$ $d\left(e_{1}, e_{3}\right)$. Since $d\left(e_{1}, e_{2}\right) \geq 0$, the inequality (4.4) holds true.

(b) If $\eta\left(e_{1}\right)<\eta\left(e_{2}\right)=\eta\left(e_{3}\right)$, then the definition of $d$ implies that $d\left(e_{1}, e_{2}\right)=$ $d\left(e_{1}, e_{3}\right)$. Since $d\left(e_{2}, e_{3}\right) \geq 0$, the inequality (4.4) holds true. 
(III) All of $\eta\left(e_{1}\right), \eta\left(e_{2}\right), \eta\left(e_{3}\right)$ are the same. In this case it is impossible to have all of $\zeta\left(e_{1}\right), \zeta\left(e_{2}\right), \zeta\left(e_{3}\right)$ be different. Again, using the fact that the inequality (4.4) is symmetric in $e_{1}=\left(g_{1}, x_{1}, n_{1}\right), e_{3}=\left(g_{3}, x_{3}, n_{3}\right)$, we can without loss of generality assume that $x_{1} \leq x_{3}$. In the following we assume that $e_{2}=\left(g_{2}, x_{2}, n_{2}\right)$.

(a) Exactly two of $\zeta\left(e_{1}\right), \zeta\left(e_{2}\right), \zeta\left(e_{3}\right)$ are the same. The following are the possible cases.

(i) If $\zeta\left(e_{1}\right)=\zeta\left(e_{2}\right) \neq \zeta\left(e_{3}\right)$, then the definition of $d$ implies that $d\left(e_{1}, e_{2}\right)=2 g_{1}, d\left(e_{2}, e_{3}\right)=g_{1}$, and $d\left(e_{1}, e_{3}\right)=g_{1}$, and inequality (4.4) becomes the trivial inequality

$$
3 g_{1} \geq g_{1}
$$

(ii) If $\zeta\left(e_{2}\right)=\zeta\left(e_{3}\right) \neq \zeta\left(e_{1}\right)$, then the definition of $d$ implies that $d\left(e_{1}, e_{2}\right)=g_{1}, d\left(e_{2}, e_{3}\right)=2 g_{2}$, and $d\left(e_{1}, e_{3}\right)=g_{1}$, and inequality (4.4) becomes the trivial inequality

$$
3 g_{1} \geq g_{1}
$$

(iii) If $\zeta\left(e_{1}\right)=\zeta\left(e_{3}\right) \neq \zeta\left(e_{2}\right)$, then the definition of $d$ implies that $d\left(e_{1}, e_{2}\right)=g_{1}, d\left(e_{2}, e_{3}\right)=g_{1}$, and $d\left(e_{1}, e_{3}\right)=2 g_{1}$, and inequality (4.4) becomes the trivial inequality

$$
2 g_{1} \geq 2 g_{1}
$$

(b) All of $\zeta\left(e_{1}\right), \zeta\left(e_{2}\right), \zeta\left(e_{3}\right)$ are the same. This situation is trivial, since each of the three distances in (4.4) are either zero or equal to exactly $2 g_{1}$.

Informally we think of the space $E$ as illustrated below:

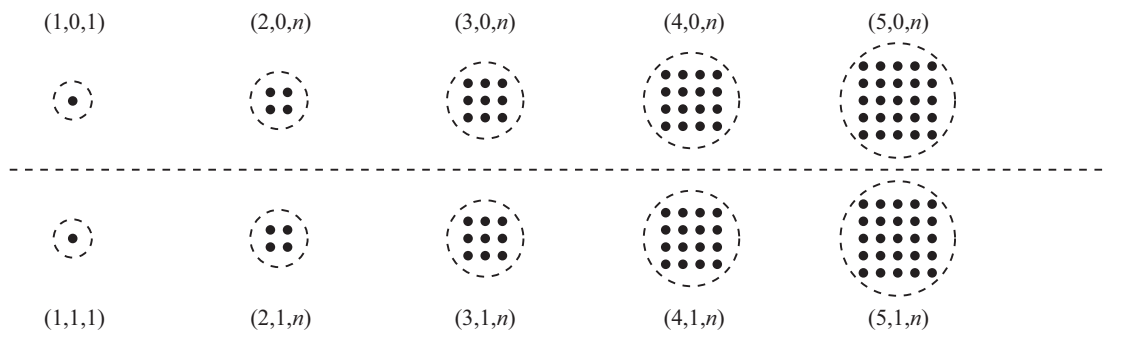

That is, the space $E$ is organized into collections of points according to the first two coordinates; the distance between points in the same collection is exactly twice the first coordinate; the distance between points in the different collections with the same first coordinates is exactly equal to the first coordinate.

Lemma 4.5. The space $(E, d)$ is a space with bounded geometry. That is, given the radius $r$, the number of elements contained in the ball of radius $r$ is at most $\frac{1}{3}(r+1)(r+2)(2 r+3)$.

Proof. The bound follows immediately from the definition of distance function $d$ in (4.3).

Lemma 4.6. The space $(E, d)$ has generalized roundness $q$ for only $q=0$. 
Proof. We consider the sequence of $2 n$-double simplices $\left[a_{i}^{k}, b_{i}^{k}\right]_{i=1}^{n}$ with

$$
a_{i}^{k}=(k, 0, i), \quad b_{i}^{k}=(k, 1, i) .
$$

We note that

and

$$
d\left(a_{i}^{k}, b_{j}^{k}\right)=k, \quad i=1, \ldots, n
$$

$$
d\left(a_{i}^{k}, a_{j}^{k}\right)=d\left(b_{i}^{k}, b_{j}^{k}\right)=2 k, \quad \text { for } i \neq j
$$

If space $(E, d)$ has generalized roundness $q \geq 0$, then the following inequality must be satisfied:

$$
\sum_{1 \leq i<j \leq n}\left[d^{q}\left(a_{i}^{k}, a_{j}^{k}\right)+d^{q}\left(b_{i}^{k}, b_{j}^{k}\right)\right] \leq \sum_{i, j=1}^{n} d^{q}\left(a_{i}^{k}, b_{j}^{k}\right)
$$

which in our case is $n(n-1) \cdot(2 k)^{q} \leq n^{2} k^{q}$. Resolving the inequality for $q$ we arrive at

$$
q \leq \log _{2}[n /(n-1)]
$$

Since $\lim _{n \rightarrow \infty} \log _{2}[n /(n-1)]=0$ we immediately see that $(E, d)$ has generalized roundness at most 0 .

Lemma 4.7. The space $(E, d)$ is coarsely round with roundness function

$$
\sigma\left(e_{1}, e_{2}\right)=\left\|\chi\left(e_{2}\right)-\chi\left(e_{1}\right)\right\|^{2}
$$

where $\chi$ is the coarse embedding of $E$ into $\mathbb{R}^{3}$ defined by

$$
\chi(e)=\chi(g, x, n)=\left((-1)^{x} \pi_{1}(n, g), \pi_{2}(n, g), \pi_{3}(g)\right)
$$

with

and

$$
\pi_{1}(n, g)=g+2 g \cdot(n \bmod g)
$$

and

$$
\pi_{2}(n, g)=g+2 g \cdot[[n / g]]
$$

$$
\pi_{3}(g)=\sum_{j=1}^{g} j^{2}=\frac{g(g+1)(2 g+1)}{6}
$$

We used $(n \bmod g)$ to denote the remainder of integer division of $n$ by $g$; and $[[n / g]$ to denote the quotient of integer division of $n$ by $g$.

The roundness function $\sigma$ is controlled by

$$
\rho_{1}(t)=t^{2}, \quad \rho_{2}(t)=5 t^{2}
$$

Proof. A short description of embedding $\chi$ is in order. The embedding has a rather simple geometric idea behind it. We use $\left(\xi_{1}, \xi_{2}, \xi_{3}\right)$ for coordinates in $\mathbb{R}^{3}$. For each fixed value of $g$ all elements $e=(g, x, n)$ are embedded into the plane $\xi_{3}=\pi_{3}(g)$ so that the distance between the planes corresponding to values of $g$ and $g-1$ is exactly $g^{2}$ units. Continuing with the same fixed value of $g$, the elements of the form $(g, 0, n)$ and $(g, 1, n)$ form a lattice by filling in centers of squares with sides of $2 g$ units, and the entire lattice occupies a rectangle stretching from $-4 g^{2}$ to $4 g^{2}$ on $\xi_{1}$ axis, and stretching from 0 to $4 g^{2}$ on $\xi_{2}$ axis.

The embedding $\chi$ by construction does not decrease distances, hence the the smaller controlling functions is $\rho_{1}(t)=t^{2}$. The largest increase of a distance by $\chi$ is found by considering the opposite corners of the lattice for fixed value of $\xi_{3}=g$. The distance between the two opposite corners is at most $\sqrt{4 g^{2}+g^{2}}$, hence the 
value of $\sigma$ for the two points is $5 g^{2}$, while the distance between two such elements in the original space is $g$. From this we obtain $\rho_{2}(t)=5 t^{2}$.

We summarize some of the properties of the space $E$ in the following proposition

Proposition 4.2. Metric space $(E, d)$ is an example of a space with the following properties:

(i) $E$ is coarsely round metric space,

(ii) E coarsely embeds into a Hilbert space,

(iii) E has generalized roundness 0 ,

(iv) $E$ is a space with bounded geometry, hence it is locally finite.

\section{REFERENCES}

[1] Dranishnikov, A.N., Gong, G., Lafforgue, V., and Yu, G. Uniform embeddings into hilbert space and a question of gromov, Canadian Mathematical Bulletin, 45(2002), no. 1, 60-70.

[2] Enflo, P., On a problem of Smirnov, Arkiv för Matematik, 8 (1970), no. 2, 107-109.

[3] Ferry, S.C., Ranicki, A. and Rosenberg, J., Novikov conjectures, index theorems and rigidity: Oberwolfach 1993, vol. 1, Cambridge Univ Pr, 1995.

[4] Gromov, M., Geometric group theory, Asymptotic invariants of infinite groups, vol. 2, Cambridge University Press, vol. 182, 1993.

[5] Lafont, J.F., and Prassidis, S., Roundness properties of groups, Geometriae Dedicata, $117(2006)$, no. $1,137-160$.

[6] Lennard, C.J., Tonge, A.M., and Weston, A., Generalized roundness and negative type, Michigan Math. J, 44(1997), no. 1, 37-45.

[7] Nowak, P.W., Coarse embeddings of metric spaces into banach spaces, Proceedings of the American Mathematical Society, 133(2005), no. 9, 2589-2596.

[8] Ostrovskii, M.I., Coarse embeddability into Banach spaces, Topology Proc., 33 (2009), 163183.

[9] Ostrovskii, M.I., Expansion properties of metric spaces not admitting a coarse embedding into a Hilbert space, C. R. Acad. Bulgare Sci. 62(2009), no. 4, 415-420.

[10] Roe, J., Lectures on coarse geometry, Amer Mathematical Society, vol. 31, 2003.

[11] Yu, G., The coarse baum-connes conjecture for spaces which admit a uniform embedding into hilbert space, Inventiones Mathematicae, 139(2000), no. 1, 201-240.

Department of Mathematics, Statistics and Computer Science,, University OF WisconsinStout, Menomonie, WI 54751

E-mail address: basyrova@uwstout.edu

Department of Mathematics, Statistics and Computer Science, University OF WisconsinStout, Menomonie, WI 54751

E-mail address: horakmatt@gmail.com 